\title{
PSA and Beyond: The Past, Present, and Future of Investigative Biomarkers for Prostate Cancer
}

\author{
Jeffrey Tosoian and Stacy Loeb* \\ The James Buchanan Brady Urological Institute, Johns Hopkins Medical Institutions, \\ Baltimore, $M D$ \\ E-mail: i⿺@jhmi.edu, stacyloeb@gmail.com
}

Received July 30, 2010; Revised September 4, 2010, Accepted September 7, 2010; Published October 1, 2010

The discovery of prostate-specific antigen (PSA) as a biomarker represented a major discovery in the early diagnosis and monitoring of prostate cancer. However, the use of PSA is limited by the lack of specificity and an inability to differentiate indolent from lifethreatening disease reliably at the time of diagnosis. A multitude of studies have aimed to improve the performance of PSA as well as identify additional biomarkers. The purpose of this study is to review available data on prostate cancer biomarkers for prostate cancer screening and prognostication, including prostatic acid phosphatase, PSA, PSA derivatives (PSA density, free PSA, pro PSA, and PSA kinetics), PCA3, GSTP1, AMACR, and other newly emerging molecular and genetic markers.

KEYWORDS: prostate cancer, biomarkers, screening, diagnosis, prostatic acid phosphatase, prostate-specific antigen (PSA), PSA density, free PSA, PSA kinetics, PSA isoforms, PCA3, GSTP-1, AMACR, single nucleotide polymorphism (SNP)

\section{HUMAN PROSTATIC ACID PHOSPHATASE}

In 1935, Kutscher and Wolbergs discovered an acid phosphatase that was present in high amounts in the human prostate[1]. A subsequent study by Gutman et al. revealed a relationship between this prostatic acid phosphatase (PAP) and prostate cancer, showing that serum PAP levels were elevated in men with metastatic disease[2]. This group additionally demonstrated that acid phosphatase activity was increased at sites of prostate cancer metastases in bone[3]. As a result of these early studies, PAP was acknowledged as the first known biomarker for prostate cancer[4].

The significance of discovering this marker was described by Huggins and Hodges, who used PAP activity to indicate the success or failure of hormonal therapy[5]. Later studies supported the use of PAP in combination with clinical findings to predict outcomes[6,7], but attempts to use the enzyme in diagnosis remained largely unsuccessful[8]. As such, the diagnosis of prostate cancer remained a purely clinical endeavor and serum levels of PAP were considered only after diagnosis.

With the introduction of prostate-specific antigen (PSA) in the 1980s, PAP was rendered largely obsolete. In addition to improved specificity, PSA was shown to have greater utility in screening and prognostication[9]. More recently, however, there has been renewed interest in serum PAP as a possible 
prognostic marker[10]. In one recent study of men with higher-risk prostate cancer, pretreatment PAP outperformed PSA and Gleason score in predicting cancer-specific survival after treatment[11]. With further investigation in a contemporary setting, PAP may ultimately prove useful in specific clinical contexts.

\section{PSA}

Secreted by prostatic epithelial cells, PSA is a 33-kDa glycoprotein that functions in the liquefaction of seminal fluid. PSA was originally described by Hara et al. in forensic studies as a marker for human semen[12]. It was later found to be present in normal benign hypertrophic and malignant prostatic tissue[13]. Studies from the Roswell Park Memorial Institute demonstrated that PSA was detectable in human serum and elevated in men with prostate cancer[14,15]. By the mid-1980s, evidence emerged that PSA was superior to PAP in monitoring prostate cancer after treatment[16], and PSA testing was approved by the Food and Drug Administration for use in this setting. Multiple studies demonstrated that PSA was a sensitive marker for detecting residual disease after treatment and tumor recurrence during follow-up[17,18]. An undetectable level of PSA after radical prostatectomy is used today to indicate the absence of recurrence[19] and serial PSA measurements are also used in the contemporary setting to define recurrence after definitive radiation therapy[20].

Beginning in the early 1990s, multiple studies suggested that serum PSA may be useful as a tool in prostate cancer screening[21,22,23]. In a multicenter clinical trial of $6,630 \mathrm{men}$, Catalona et al. reported that PSA, when used in conjunction with digital rectal examination (DRE), enhanced early prostate cancer detection[24]. These and other findings led to FDA approval of PSA for prostate cancer screening, using a threshold of $4.0 \mathrm{ng} / \mathrm{ml}$.

The introduction of large-scale PSA-based screening was associated with dramatic increases in prostate cancer incidence throughout the 1990s[25]. Moreover, a greater proportion of men were diagnosed with early-stage disease and far fewer presented with distant metastases[26]. This "stage migration" is well documented in the Surveillance, Epidemiology, and End Results (SEER) database, wherein the proportion of men with metastases at presentation decreased from 16 to $4 \%$ with screening[27]. Indeed, a large analysis by Jemal et al. suggested that widespread PSA testing was associated with decreased incidence of late-stage disease and prostate cancer-specific mortality[28].

Using $4.0 \mathrm{ng} / \mathrm{ml}$ as the threshold for biopsy, Catalona et al. reported that a significant proportion of cancers had spread to the prostate capsule by the time of detection[29]. Furthermore, longitudinal analysis revealed that of men with PSA $\geq 2.5 \mathrm{ng} / \mathrm{ml}$, approximately one-half demonstrated a PSA increase to 4.0 $\mathrm{ng} / \mathrm{ml}$ within 4 years[30]. As such, the authors decreased the threshold for biopsy to $2.5 \mathrm{ng} / \mathrm{ml}$ in their screening study[31]. Later, they demonstrated a direct relationship between PSA levels at diagnosis and the likelihood of organ-confined disease[32]. In men with preoperative PSA levels of 2.6-4.0, 4.1-7.0, 7.1-10.0, and $>10 \mathrm{ng} / \mathrm{ml}$, the rates of organ-confined disease were $81,74,72$, and $60 \%$, respectively, and 10-year recurrence-free survival estimates differed significantly across preoperative PSA strata (log-rank test, $p=0.0001$ ). This study provided evidence that cancers detected at PSA levels of $2.6-4.0 \mathrm{ng} / \mathrm{ml}$ have higher rates of organ-confined disease and improved 10-year recurrence-free survival than those detected at higher PSA levels.

Additional trials provided support for the use of a lower PSA threshold for biopsy. In 2004, Thompson and colleagues discovered cancer in 17\% of men with low PSA (1.1-2.0 ng/ml) and a normal DRE, indicating that even the most stringent biopsy criteria would miss a significant proportion of cancers[33]. Alarmingly, this trial demonstrated high-grade disease in $14.9 \%$ of the cancers detected at a PSA $\leq 4.0 \mathrm{ng} / \mathrm{ml}$.

A problem with the use of a lower PSA threshold for biopsy is that PSA may be elevated as a result of various nonmalignant conditions, e.g., benign prostatic hyperplasia and prostatitis, or after iatrogenic manipulation of the prostate, such as transrectal ultrasound[34]. These confounders may lead to unnecessary biopsies and this problem would be compounded by lowering PSA thresholds. Even using 
the conventional threshold, $75 \%$ of men with PSA $4.0-10.0 \mathrm{ng} / \mathrm{ml}$ who undergo biopsy do not have cancer, introducing a significant source of unnecessary cost, as well as potential anxiety and morbidity[35]. At the same time, studies have indicated that PSA screening leads to the detection of some cancers that would have otherwise remained undetected during life[36]. This is concerning, as unnecessary treatment of indolent cancers may be associated with significant morbidity, especially in older men[37].

There has been a great deal of investigation aimed at improving the accuracy of PSA-based screening. Adjunctive measurements considering the rate of PSA changes with time, the ratios of free and proteinbound PSA, and the relationship of PSA to prostate size have improved performance characteristics in some settings[38]. Several nomograms combining PSA with other clinical variables have also been developed to improve prognostic value beyond that of individual tests[39]. At the same time, there has been increased interest in active surveillance programs for low-risk patients, presenting an option to reduce the extent of unnecessary overtreatment[40,41].

Amid the many questions surrounding PSA, a most fundamental question remains: Is PSA-based screening beneficial? Despite the evidence presented above and elsewhere, this question came under increased scrutiny when two large trials demonstrated contradictory findings. Preliminary results of a U.S. trial comparing annual screening to usual care found no significant difference in cause-specific mortality[42], although there were numerous methodological limitations to this study. Conversely, in a large European trial, Schroder et al. demonstrated a $20 \%$ reduction in cause-specific mortality in the screened arm vs. the control arm[43]. Considering these findings in combination with the available literature, PSA-based screening has undoubtedly contributed to the significant decline in prostate cancermortality rates observed in the U.S. and abroad since the late 1990s[44,45]. Nonetheless, there is continued debate as to whether the benefits of prostate cancer screening outweigh its risks.

\section{PSA DERIVATIVES}

\section{Free PSA}

Serum PSA circulates in either an unbound "free" form or bound to one of several proteins, most frequently alpha-1-antichymotrypsin (ACT)[46,47]. Levels of free PSA (fPSA) can be detected and compared to total PSA, yielding the proportion of free PSA (\%fPSA). Studies have shown that men with the highest proportions of complexed PSA are more likely to have prostate cancer[48] and that \%fPSA is lower in men with prostate cancer as compared to benign prostatic hyperplasia (BPH)[49]. Thus, \%fPSA showed promise in distinguishing malignant from benign prostate disease.

In addition to investigating the function of fPSA, follow-up studies helped to establish its performance characteristics. In 1995, Prestigiacamo and Stamey demonstrated a median \%fPSA of 8.9 in men diagnosed with prostate cancer and 16.5 in men with BPH[50]. In the same year, Luderer et al. published a comparative study including men with prostate cancer, benign prostate disease, as well as healthy asymptomatic controls[51]. Among all subjects, both total PSA and \%fPSA differentiated cancer from benign conditions. Furthermore, \%fPSA outperformed total PSA in men in the "diagnostic gray zone" (PSA $=4.0-10.0 \mathrm{ng} / \mathrm{ml})$. Using a threshold $\mathrm{fPSA}$ value of $25 \%$, another study of men with intermediate PSA levels yielded 95\% sensitivity and 20\% specificity for prostate cancer diagnosis[52]. When restricted to men with total PSA of $3.0-7.0 \mathrm{ng} / \mathrm{ml}$, the use of a $20 \%$ fPSA threshold improved specificity to $38 \%$.

One potential benefit of using \%fPSA is reducing the proportion of unnecessary biopsies. In 1998, a prospective multicenter trial showed that \%fPSA reduced unnecessary biopsies by $20 \%$ when using a threshold fPSA of 25\%[53]. This trial also showed that, in general, cancers detected at fPSA $>25 \%$ were smaller and lower grade. Other trials similarly demonstrated a reduction in the unnecessary biopsy rate, most commonly using \%fPSA thresholds ranging from 20 to 27\%[31]. In light of these data, fPSA was 
approved by the FDA for use in the screening and diagnosis of prostate cancer at PSA levels between 4.0 and $10.0 \mathrm{ng} / \mathrm{ml}$.

Numerous studies have explored methods of optimizing diagnostic accuracy of fPSA. One such study demonstrated a higher \%fPSA in larger prostates, suggesting clinical adjustment of threshold values based on prostate volume[54]. Subsequent trials identified other challenges to the use of fPSA, such as conditions at the time of sample obtainment, in vitro instability, and interassay variability[50,55,56]. These factors may help to explain inconsistencies in the performance of fPSA[57]. Despite known limitations, a comprehensive meta-analysis demonstrated that \%fPSA significantly outperformed total PSA in predicting biopsy outcomes in men with intermediate PSA[58]. Considering the available data, fPSA appears to be a useful tool for diagnosis, particularly in men with intermediate levels of serum PSA.

\section{PSA Density}

To enhance the diagnostic accuracy of PSA, Benson et al. described the concept of PSA density (PSAD) as the ratio of PSA concentration to prostate volume[59]. This initial study found significant differences in mean PSAD between men with prostate cancer vs. BPH $(0.581,0.044 ; p<0.002)$, identifying the potential utility of PSAD in differentiating prostate cancer from benign disease. Subsequent studies evaluated PSAD in additional clinical settings, with many demonstrating a modest improvement in diagnostic accuracy when PSAD was considered in addition to PSA[60,61].

Because the transition zone is primarily involved in $\mathrm{BPH}$, subsequent studies investigated the value of adjusting PSA to transition zone volume rather than total prostate volume. A study by Kalish et al. found that PSA density of the transition zone (PSADTZ) was more predictive of positive biopsy than conventional PSAD in patients with intermediate PSA levels $(4.0-10.0 \mathrm{ng} / \mathrm{ml})$ [62]. Kikuchi et al. similarly demonstrated that PSADTZ was superior to PSAD in distinguishing cancer from BPH[63]. Despite these positive findings, other studies concluded that PSAD had value only in limited patient subgroups, and still others failed to identify a single practical use for PSAD or PSADTZ that improved predictive performance in screening[64,65].

Other studies have demonstrated the utility of PSAD in predicting clinicopathological features of disease, such as Gleason score and total cancer volume at radical prostatectomy[66]. Similarly, on multivariate analysis, Radwan et al. found that PSAD independently predicted positive surgical margins, extracapsular extension, seminal vesicle invasion, and biochemical failure after prostatectomy[67]. These findings supported earlier data suggesting that PSAD could predict tumor pathology in men with nonpalpable prostate cancer[68]. More recently, Tseng et al. demonstrated that PSA density may aid in predicting the risk of progression in men on active surveillance[69]. In the contemporary setting, PSAD may be most effective when used with other clinical factors to stratify risk and weigh treatment options. A drawback of PSAD is the need for transrectal ultrasound, which often is not performed as a separate procedure prior to prostate biopsy, as well as limitations in the accuracy and interexaminer variability in prostate size measurement.

\section{PSA Isoforms}

fPSA exists in multiple molecular isoforms, including a BPH-related isoform termed BPSA[70,71], inactive PSA (iPSA), and proPSA, which has been associated with prostate cancer[74,75]. ProPSA was originally described with a 7-amino-acid leader sequence and was subsequently found to circulate in other forms with leader sequences of 5, 4, and 2 amino acids[72,73].

In 2003, a study of men with total PSA levels of $2.0-10.0 \mathrm{ng} / \mathrm{ml}$ demonstrated that the percentage of proPSA (\%pPSA), calculated as proPSA divided by fPSA, was more specific for detecting prostate cancer when compared to complexed PSA or fPSA alone[76]. In men with PSA of 2.5-4.0 ng/ml, Sokoll et al. similarly showed \%pPSA outperformed \% fPSA in detecting prostate cancer and reducing unnecessary 
biopsies[77]. Subsequently, the focus shifted to the [-2]proPSA (p2PSA) isoform, which was recently tested in a large European population[78]. Other studies have shown that in men with PSA $\leq 10.0 \mathrm{ng} / \mathrm{ml}$, the proportion of p2PSA (\%p2PSA) significantly outperformed \%fPSA in diagnosing prostate cancer[79,80].

In addition to improving diagnostic accuracy, one subsequent trial revealed that \%pPSA selected for higher-grade disease and extracapsular extension[81]. More recent investigations of \%pPSA have had similarly encouraging results. For example, a study of men with low-risk prostate cancer in active surveillance demonstrated that levels of serum and tissue proPSA at presentation were significantly associated with progression to unfavorable biopsy findings during follow-up[82]. Thus, proPSA may be useful in the surveillance setting to predict men who will eventually require curative intervention.

More recently, proPSA has been incorporated into a proprietary equation known as the Beckman Coulter Prostate Health Index (phi), which is calculated as: (p2PSA pg/ml / fPSA ng/ml) $\times\left(\right.$ PSA ng/ml) ${ }^{1 / 2}$. In one study, phi offered the greatest discrimination for prostate cancer on receiver operating characteristic analysis. Additional investigation on the role of proPSA and phi are currently underway.

\section{PSA Kinetics}

The calculation of changes in PSA over time was first described by Carter et al. using data from the Baltimore Longitudinal Study of Aging[83]. Using comprehensive longitudinal data, Carter and colleagues revealed greater increases in PSA with time (PSA velocity, PSAV) in men who were eventually diagnosed with prostate cancer when compared to men who were never diagnosed with prostate cancer. Importantly, these differences in PSAV were observed several years prior to clinical diagnosis, conferring the potential for very early identification of disease. Meanwhile, serum PSA levels at this time did not differentiate between the groups. Using a cutoff of $0.75 \mathrm{ng} / \mathrm{ml} / \mathrm{year}$, PSAV distinguished men with prostate cancer from BPH and healthy controls with a specificity of 90 and $100 \%$, respectively. Further analysis revealed that longitudinal PSA measurements followed an exponential growth pattern in men who were later diagnosed with cancer[84]. This shift in growth curves occurred at an average of 7-9 years prior to clinical diagnosis, also suggesting a role of PSAV in early detection.

Another method of quantifying changes in PSA over time, PSA doubling-time (PSADT), is defined as the time it takes for serum PSA to double. Initial studies of PSADT demonstrated its utility in characterizing the biological significance of disease recurrence after treatment. In 1999, Pound et al. demonstrated that a postoperative PSADT <10 months predicted worse metastasis-free survival[19]. Follow-up studies demonstrated that lower PSADT was associated with increased risk of cancer-specific mortality in men with PSA recurrence after surgery [85] and radiation therapy[86].

Many recent studies have examined the role of PSA kinetics in prognostication. One such study revealed that PSAV predicts the likelihood of clinically significant cancer in men with PSA levels $\leq 4.0$ $\mathrm{ng} / \mathrm{ml}$ [87]. Another study in men with normal PSA levels demonstrated an association between PSAV and long-term prognosis. In this analysis, Carter et al. showed that PSAV measured 10-15 years prior to prostate cancer diagnosis was associated with rates of cancer-specific mortality 25 years later[88]. Two significant studies by D'Amico et al. showed that pretreatment PSAV $>2 \mathrm{ng} / \mathrm{ml} / \mathrm{year}$ was associated with increased prostate cancer-specific mortality after either radiation therapy or radical prostatectomy[89,90].

Despite its success in predicting prognosis, the utility of PSA kinetics in screening has become increasingly controversial[91]. In 2006, Thompson et al. reported that PSA kinetics did not reliably predict a diagnosis of prostate cancer in men with PSA $\leq 4.0 \mathrm{ng} / \mathrm{ml}$ [92]. Similarly, a study of 1,699 men with PSA levels $<10.0 \mathrm{ng} / \mathrm{ml}$ demonstrated that PSADT and PSAV had little to no value in predicting biopsy results[93]. Furthermore, data from the European Randomized Study of Screening for Prostate Cancer (ERSPC) demonstrated that PSAV was not an independent predictor of positive biopsy[94]. Thus, some critics have concluded that despite the prognostic value, PSA kinetics have little value in predicting biopsy results[95,96,97]. 
Ultimately, the inconsistencies of PSA kinetics may be partially explained by differences in methodology. For example, predictive performance can vary greatly depending on data points used in calculating kinetic values. One study recommended using at least three measurements obtained over 2 years for accurate calculation[98]. Kinetic values based on measurements obtained in the short term (e.g., $<6$ months apart) may be less useful due to individual fluctuations in serum PSA levels[99]. Furthermore, differences in PSA assay standardization may also affect calculations in the real-world setting[100]. In an effort to standardize their use and optimize predictive ability, there has recently been greater attention focused on data collected for kinetic studies[101]. Nonetheless, the concept of PSA kinetics has improved the prognostic value of longitudinal data beyond the capabilities of PSA measurements alone.

\section{PCA3}

In 1999, Bussemakers et al. described DD3 (also known as PCA3) as a potential biomarker for prostate cancer[102]. Additional studies from this group characterized DD3 as a noncoding, prostate-specific mRNA that is highly overexpressed in prostate cancer[103]. Initial studies demonstrated high sensitivity and specificity for prostate cancer, as DD3 was overexpressed in $94.6 \%$ of cancerous tissue samples and not in controls. Follow-up studies corroborated DD3 as a highly specific tissue marker, revealing insignificant DD3 expression in benign tissue and in nonprostatic tumors. Importantly, mRNA was expressed at 34-fold increased levels in malignant vs. nonmalignant tissue.

In a subsequent study, Hessels et al. used RT-PCR to demonstrate a 66-fold increase of DD3 expression in prostate tumors[104]. RT-PCR was then used to detect DD3 in urine, revealing a sensitivity of $67 \%$ and a negative predictive value of $90 \%$. In a more recent cohort study, Marks et al. found that DD3 was superior to PSA in predicting prostate cancer in men with elevated PSA and a previous negative biopsy[105]. At the same time, there is evidence that the PCA3 score is associated with clinical and pathological features. Urine PCA3 scores demonstrated a significant association with extracapsular extension, tumor volume, and Gleason score[106,107]. A relationship between PCA3 and Gleason score was similarly demonstrated in a European population[108].

With a better understanding of its clinical strengths and limitations, PCA3 may serve as a valuable biomarker moving forward. Observed associations with pathologic tumor features suggested a potential role in selecting or monitoring men with low-risk cancers. As such, our institution recently assessed the relationship of urinary PCA3 and repeat biopsy results in an active surveillance population[109]. Analysis revealed higher PCA3 scores in men with high-risk cancer on surveillance biopsy, particularly in those upgraded based on Gleason score, but differences were not statistically significant. Furthermore, a sensitive and specific PCA3 threshold for biopsy could not be identified due to considerable overlap of PCA3 scores between groups. Although its role in surveillance remains unclear, PCA3 has shown great promise in the general population as a diagnostic and prognostic marker for prostate cancer.

\section{GSTP1}

Enzymes of the glutathione S-transferase (GST) family have many functions in cellular metabolism, notably including the detoxification of potentially harmful substrates[110]. In 1994, Lee et al. demonstrated hypermethylation of promoter sequences at the GSTP1 gene in human prostatic cancer tissue specimens[111]. Additionally, the authors observed a substantial decrease in GSTPI expression associated with prostatic cancers, in contrast to abundant GSTP1 expression in normal prostatic epithelium. These and other studies led to the hypothesis that GSTPl-encoded enzymes serve as "caretakers" of prostatic cells, and that failure to express these enzymes due to somatic CpG hypermethylation plays a role in prostatic carcinogenesis[112]. Nonetheless, GSTP1 promoter hypermethylation has been observed in $>90 \%$ of prostatic cancers, making it the most common DNA alteration associated with prostate cancer[112,113,114]. 
Because tumor DNA can be detected in the bodily fluids of some prostate cancer patients[115,116], Goessl et al. utilized methylation-specific PCR to evaluate GSTP1 promoter hypermethylation in various samples obtained from men with prostate cancer[117]. The authors observed GSTP1 promoter hypermethylation at rates of $94 \%$ in tissue, $72 \%$ in serum, $50 \%$ in ejaculate, and $36 \%$ in urine. Remarkably, hypermethylation was found in $100 \%$ of serum specimens from patients with locally advanced or metastatic disease. By comparison, hypermethylation was absent in all tissue samples and bodily fluids obtained from men with biopsy-confirmed $\mathrm{BPH}$.

DNA-based molecular tumor markers have potential for very high sensitivity and specificity, theoretically reaching $100 \%[118]$. A review of other GSTP1 methylation studies in urine revealed a consistently high specificity, ranging from 93 to $100 \%[117,119,120,121,122,123,124]$. Nevertheless, detection rates in these studies were not sufficiently reliable, ranging from 19 to $76 \%$. Prostate cancer detection rates based on plasma samples were similarly inadequate, ranging from 13 to 72\%[117,121,122]. Furthermore, very few studies have identified GSTP1 promoter hypermethylation in ejaculate, and detecting cancer biomarkers in ejaculate has traditionally been very difficult[125]. Ultimately, however, the identification of a molecular marker for prostate cancer represented a major scientific advance. With improved sensitivity, GSTP1 promoter hypermethylation may prove to be a useful marker in prostate cancer detection.

\section{AMACR}

Advances in molecular biology have allowed for the discovery of other novel biomarkers for prostate cancer. Using cDNA library subtraction and high-throughput microarray screening, $\mathrm{Xu}$ et al. demonstrated overexpression of alpha-methylacyl coenzyme A racemase (AMACR) in prostate cancer at both the mRNA and protein levels[126]. AMACR is an enzyme with a well-characterized role in the betaoxidation of branched-chain fatty acids and bile acid intermediates[127]. A potential relationship between this enzyme and prostate cancer development is particularly intriguing because the main sources of branched-chain fatty acids in humans, including beef and dairy products, have been implicated as dietary risk factors for prostate cancer[128].

In 2001, Jiang et al. assessed AMACR as a molecular biomarker for prostate cancer[129]. The authors used a monoclonal antibody to stain 137 prostatic cancer tissue samples and 70 benign prostate tissue samples. They reported positive expression of AMACR in all 137 prostate cancer specimens, and analysis revealed a sensitivity of $100 \%$ and specificity of $88 \%$. A study by Rubin et al. in 2002 similarly demonstrated overexpression of AMACR in prostate cancer[130]. In this study, AMACR expression in prostate biopsy specimens detected cancer with $97 \%$ sensitivity and $100 \%$ specificity. An additional study by Luo et al. demonstrated that AMACR and p63 antibodies could be used in combination to further enhance the accuracy of prostate cancer diagnosis[131]. The high sensitivity and specificity of AMACR staining holds great promise for making a diagnosis when conventional staining methods are inconclusive[132].

\section{Genetic Markers}

The recent advent of genome-wide association studies has revolutionized the field of prostate cancer genetics. More than 30 single nucleotide polymorphisms (SNPs) have been identified across the human genome with demonstrated associations with prostate cancer susceptibility. For example, SNPs on multiple regions of chromosome 8q24 now have well-validated associations with prostate cancer risk[133]. In conjunction with two alleles on chromosome 17, these variants were shown to have a cumulative relationship with prostate cancer risk[134]. Specifically, carriers of five risk alleles with a positive family history of prostate cancer had a 9.5-fold increased risk of prostate cancer, as compared with noncarriers, in a landmark study by Zheng et al.[134]. Many other SNPs have since been identified 
with links to prostate cancer susceptibility, although evidence has been less convincing on the relationship between many of these alleles and disease aggressiveness[135,136]. Because genetic susceptibility testing is becoming commercially available, additional study is urgently needed to determine whether these new markers have a role in prostate cancer screening and risk stratification.

\section{CONCLUSION}

The use of biomarkers for prostate cancer extends back nearly a century, but it was forever changed in the 1980s with the discovery of PSA. Serum PSA demonstrated effectiveness as a marker for recurrence after treatment and it continues to be used for this purpose today. Since the initiation of widespread PSA-based screening, most contemporary patients are diagnosed with early-stage prostate cancer that is curable with treatment. Despite this, the value of PSA in screening has come under scrutiny due to its lack of specificity and potential for the overdiagnosis of nonthreatening cancers. To improve performance characteristics, a number of PSA derivatives and isoforms have also been studied. These biomarkers have shown promising, but mixed, results.

More recently discovered biomarkers, such as DD3 (PCA3), GSTP1, and AMACR, have favorable performance characteristics in some clinical settings. In order to best utilize these markers, however, additional study is needed to define the appropriate context and optimal parameters for their use. Until the discovery of a biomarker with both high sensitivity and specificity, screening will likely continue to involve a joint consideration of multiple markers in combination with other clinical factors.

At the same time, emerging genetics discoveries may represent a new revolution in prostate cancer research. In the future, genomic testing may allow improved identification of men at risk for prostate cancer, or may help to guide the use of existing biomarkers and personalize screening protocols.

Despite numerous limitations, there have been great strides in prostate cancer biomarker research in recent years. Ongoing investigation into these and other new markers in the future will continue to improve our ability to stratify prostate cancer risk and prognosis.

\section{REFERENCES}

1. Kutscher, W. and Wolbergs, H.: (1935) Prostataphosphatase. Z. Physiol. Chem. 236, 237.

2. Gutman, A.B. and Gutman, E.B. (1938) An "acid" phosphatase occurring in the serum of patients with metastasizing carcinoma of the prostate gland. J. Clin. Invest. 17(4), 473.

3. Gutman, E.B., Sproul, E.E., and Gutman, A.B. (1936) Significance of increased phosphatase activity of bone at the site of osteoplastic metastases secondary to carcinoma of the prostate gland. Am. J. Cancer 28, 1936.

4. Burgess, C.T. and Evans, R.W. (1949) Serum acid phosphatase in the diagnosis of carcinoma of the prostate. Lancet 2, 790 .

5. Huggins, C. and Hodges, C.V. (2002) Studies on prostatic cancer: I. The effect of castration, of estrogen and of androgen injection on serum phosphatases in metastatic carcinoma of the prostate. 1941. J. Urol. 168, 9.

6. Johnson, D.E., Prout, G.R., Scott, W.W., et al. (1976) Clinical significance of serum acid phosphatase levels in advanced prostatic carcinoma. Urology 8, 123.

7. Whitesel, J.A., Donohue, R.E., Mani, J.H., et al. (1984) Acid phosphatase: its influence on the management of carcinoma of the prostate. J. Urol. 131, 70.

8. Griffiths, S. and Shaw, L.M. (1983) The laboratory diagnosis of prostatic adenocarcinoma. Crit. Rev. Clin. Lab. Sci. 19, 187.

9. Lowe, F.C. and Trauzzi, S.J. (1993) Prostatic acid phosphatase in 1993. Its limited clinical utility. Urol. Clin. North Am. 20, 589.

10. Taira, A., Merrick, G., Wallner, K., et al. (2007) Reviving the acid phosphatase test for prostate cancer. Oncology (Williston Park) 21, 1003.

11. Fang, L.C., Dattoli, M., Taira, A., et al. (2008) Prostatic acid phosphatase adversely affects cause-specific survival in patients with intermediate to high-risk prostate cancer treated with brachytherapy. Urology 71, 146.

12. Hara, M., Koyanagi, Y., Inoue, T., et al. (1971) Some physico-chemical characteristics of " -seminoprotein", an antigenic component specific for human seminal plasma. Forensic immunological study of body fluids and secretion. VII. Nihon Hoigaku Zasshi 25, 322. 
13. Wang, M.C., Valenzuela, L.A., Murphy, G.P., et al. (1979) Purification of a human prostate specific antigen. Invest. Urol. 17, 159.

14. Papsidero, L.D., Wang, M.C., Valenzuela, L.A., et al. (1980) A prostate antigen in sera of prostatic cancer patients. Cancer Res. 40, 2428.

15. Kuriyama, M., Wang, M.C., Papsidero, L.D., et al. (1980) Quantitation of prostate-specific antigen in serum by a sensitive enzyme immunoassay. Cancer Res. 40, 4658.

16. Stamey, T.A., Yang, N., Hay, A.R., et al. (1987) Prostate-specific antigen as a serum marker for adenocarcinoma of the prostate. N. Engl. J. Med. 317, 909.

17. Oesterling, J.E., Chan, D.W., Epstein, J.I., et al. (1988) Prostate specific antigen in the preoperative and postoperative evaluation of localized prostatic cancer treated with radical prostatectomy. J. Urol. 139, 766.

18. Landmann, C. and Hunig, R. (1989) Prostatic specific antigen as an indicator of response to radiotherapy in prostate cancer. Int. J. Radiat. Oncol. Biol. Phys. 17, 1073.

19. Pound, C.R., Partin, A.W., Eisenberger, M.A., et al. (1999) Natural history of progression after PSA elevation following radical prostatectomy. JAMA 281, 1591.

20. Boukaram, C. and Hannoun-Levi, J.M. (2010) Management of prostate cancer recurrence after definitive radiation therapy. Cancer Treat. Rev. 36, 91.

21. Catalona, W.J., Smith, D.S., Ratliff, T.L., et al. (1991) Measurement of prostate-specific antigen in serum as a screening test for prostate cancer. N. Engl. J. Med. 324, 1156.

22. Brawer, M.K., Chetner, M.P., Beatie, J., et al. (1992) Screening for prostatic carcinoma with prostate specific antigen. J. Urol. 147, 841.

23. Cooner, W.H., Mosley, B.R., Rutherford, C.L., Jr., et al. (1990) Prostate cancer detection in a clinical urological practice by ultrasonography, digital rectal examination and prostate specific antigen. J. Urol. 143, 1146.

24. Catalona, W.J., Richie, J.P., Ahmann, F.R., et al. (1994) Comparison of digital rectal examination and serum prostate specific antigen in the early detection of prostate cancer: results of a multicenter clinical trial of 6,630 men. J. Urol. 151, 1283. Brawley, O.W., Knopf, K., and Merrill, R. (1998) The epidemiology of prostate cancer part I: descriptive epidemiology. Semin. Urol. Oncol. 16, 187.

26. Cooperberg, M.R., Lubeck, D.P., Mehta, S.S., et al. (2003) Time trends in clinical risk stratification for prostate cancer: implications for outcomes (data from CaPSURE). J. Urol. 170, S21.

27. Public-Use, S. S. D. I.-S. R. Surveillance, Epidemiology, and End Results (SEER) Program. National Cancer Institute, DCCPS, Surveillance Research Program, Cancer Statistics Branch, released April 2006, Nov 2005 Sub (1973-2003 varying).

28. Jemal, A., Ward, E., Wu, X., et al. (2005) Geographic patterns of prostate cancer mortality and variations in access to medical care in the United States. Cancer Epidemiol. Biomarkers Prev. 14, 590.

29. Catalona, W.J., Smith, D.S., Ratliff, T.L., et al. (1993) Detection of organ-confined prostate cancer is increased through prostate-specific antigen-based screening. JAMA 270, 948.

30. Smith, D.S., Catalona, W.J., and Herschman, J.D. (1996) Longitudinal screening for prostate cancer with prostatespecific antigen. JAMA 276, 1309.

31. Catalona, W.J., Smith, D.S., and Ornstein, D.K. (1997) Prostate cancer detection in men with serum PSA concentrations of 2.6 to $4.0 \mathrm{ng} / \mathrm{mL}$ and benign prostate examination. Enhancement of specificity with free PSA measurements. JAMA 277, 1452.

32. Antenor, J.A., Roehl, K.A., Eggener, S.E., et al. (2005) Preoperative PSA and progression-free survival after radical prostatectomy for Stage T1c disease. Urology 66, 156.

33. Thompson, I.M., Pauler, D.K., Goodman, P.J., et al. (2004) Prevalence of prostate cancer among men with a prostatespecific antigen level < or =4.0 ng per milliliter. N. Engl. J. Med. 350, 2239.

34. Oesterling, J.E. (1991) Prostate specific antigen: a critical assessment of the most useful tumor marker for adenocarcinoma of the prostate. J. Urol. 145, 907.

35. Barry, M.J. (2001) Clinical practice. Prostate-specific-antigen testing for early diagnosis of prostate cancer. N. Engl. J. Med. 344, 1373.

36. Draisma, G., Boer, R., Otto, S.J., et al. (2003) Lead times and overdetection due to prostate-specific antigen screening: estimates from the European Randomized Study of Screening for Prostate Cancer. J. Natl. Cancer Inst. 95, 868.

37. Loeb, S., Roehl, K.A., Helfand, B.T., et al. (2008) Complications of open radical retropubic prostatectomy in potential candidates for active monitoring. Urology 72, 887.

38. Gretzer, M.B. and Partin, A.W. (2003) PSA markers in prostate cancer detection. Urol. Clin. North Am. $30,677$.

39. Makarov, D.V., Trock, B.J., Humphreys, E.B., et al. (2007) Updated nomogram to predict pathologic stage of prostate cancer given prostate-specific antigen level, clinical stage, and biopsy Gleason score (Partin tables) based on cases from 2000 to 2005. Urology 69, 1095.

40. Klotz, L. (2008) Active surveillance for prostate cancer: trials and tribulations. World J. Urol. $26,437$.

41. Stattin, P., Holmberg, E., Johansson, J.E. et al. (2010) Outcomes in localized prostate cancer: National Prostate Cancer Register of Sweden follow-up study. J. Natl. Cancer Inst. 102, 950. 
42. Andriole, G.L., Crawford, E.D., Grubb, R.L., $3^{\text {rd }}$, et al. (2009) Mortality results from a randomized prostate-cancer screening trial. N. Engl. J. Med. 360, 1310.

43. Schroder, F.H., Hugosson, J., Roobol, M.J., et al. (2009) Screening and prostate-cancer mortality in a randomized European study. N. Engl. J. Med. 360, 1320.

44. Edwards, B.K., Ward, E., Kohler, B.A., et al. Annual report to the nation on the status of cancer, 1975-2006, featuring colorectal cancer trends and impact of interventions (risk factors, screening, and treatment) to reduce future rates. Cancer 116, 544.

45. Baade, P.D., Youlden, D.R., and Krnjacki, L.J. (2009) International epidemiology of prostate cancer: geographical distribution and secular trends. Mol. Nutr. Food Res. 53, 171.

46. Christensson, A., Laurell, C.B., and Lilja, H. (1990) Enzymatic activity of prostate-specific antigen and its reactions with extracellular serine proteinase inhibitors. Eur. J. Biochem. 194, 755.

47. Lilja, H., Christensson, A., Dahlen, U., et al. (1991) Prostate-specific antigen in serum occurs predominantly in complex with alpha 1-antichymotrypsin. Clin. Chem. 37, 1618.

48. Stenman, U.H., Leinonen, J., Alfthan, H., et al. (1991) A complex between prostate-specific antigen and alpha 1antichymotrypsin is the major form of prostate-specific antigen in serum of patients with prostatic cancer: assay of the complex improves clinical sensitivity for cancer. Cancer Res. 51, 222.

49. Christensson, A., Bjork, T., Nilsson, O., et al. (1993) Serum prostate specific antigen complexed to alpha 1antichymotrypsin as an indicator of prostate cancer. J. Urol. 150, 100.

50. Prestigiacomo, A.F. and Stamey, T.A. (1995) Clinical usefulness of free and complexed PSA. Scand. J. Clin. Lab. Invest. Suppl. 221, 32.

51. Luderer, A.A., Chen, Y.T., Soriano, T.F., et al. (1995) Measurement of the proportion of free to total prostate-specific antigen improves diagnostic performance of prostate-specific antigen in the diagnostic gray zone of total prostatespecific antigen. Urology 46, 187.

52. Partin, A.W., Brawer, M.K., Subong, E.N., et al. (1998) Prospective evaluation of percent free-PSA and complexedPSA for early detection of prostate cancer. Prostate Cancer Prostatic Dis. 1, 197.

53. Catalona, W.J., Partin, A.W., Slawin, K.M., et al. (1998) Use of the percentage of free prostate-specific antigen to enhance differentiation of prostate cancer from benign prostatic disease: a prospective multicenter clinical trial. JAMA 279, 1542.

54. Catalona, W.J., Smith, D.S., Wolfert, R.L., et al. (1995) Evaluation of percentage of free serum prostate-specific antigen to improve specificity of prostate cancer screening. JAMA 274, 1214.

55. Lilja, H., Haese, A., Bjork, T., et al. (1999) Significance and metabolism of complexed and noncomplexed prostate specific antigen forms, and human glandular kallikrein 2 in clinically localized prostate cancer before and after radical prostatectomy. J. Urol. 162, 2029.

56. Piironen, T., Pettersson, K., Suonpaa, M., et al. (1996) In vitro stability of free prostate-specific antigen (PSA) and prostate-specific antigen (PSA) complexed to alpha 1-antichymotrypsin in blood samples. Urology 48, 81.

57. Djavan, B., Remzi, M., Schulman, C.C., et al. (2002) Repeat prostate biopsy: who, how and when?. a review. Eur. Urol. 42, 93.

58. Roddam, A.W., Duffy, M.J., Hamdy, F.C., et al. (2005) Use of prostate-specific antigen (PSA) isoforms for the detection of prostate cancer in men with a PSA level of 2-10 ng/ml: systematic review and meta-analysis. Eur. Urol. 48, 386.

59. Benson, M.C., Whang, I.S., Pantuck, A., et al. (1992) Prostate specific antigen density: a means of distinguishing benign prostatic hypertrophy and prostate cancer. J. Urol. 147, 815.

60. Ohori, M., Dunn, J.K., and Scardino, P.T. (1995) Is prostate-specific antigen density more useful than prostatespecific antigen levels in the diagnosis of prostate cancer? Urology 46, 666.

61. Egawa, S., Suyama, K., Takashima, R., et al. (1999) Prospective evaluation of prostate cancer detection by prostatespecific antigen-related parameters. Int. J. Urol. 6, 493.

62. Kalish, J., Cooner, W.H., and Graham, S.D., Jr. (1994) Serum PSA adjusted for volume of transition zone (PSAT) is more accurate than PSA adjusted for total gland volume (PSAD) in detecting adenocarcinoma of the prostate. Urology 43, 601.

63. Kikuchi, E., Nakashima, J., Ishibashi, M., et al. (2000) Prostate specific antigen adjusted for transition zone volume: the most powerful method for detecting prostate carcinoma. Cancer 89, 842.

64. Lujan, M., Paez, A., Llanes, L., et al. (2001) Prostate specific antigen density. Is there a role for this parameter when screening for prostate cancer? Prostate Cancer Prostatic Dis. 4, 146.

65. Giannarini, G., Scott, C. A., Moro, U., et al. (2008) Are PSA density and PSA density of the transition zone more accurate than PSA in predicting the pathological stage of clinically localized prostate cancer? Urol. Oncol. $26,353$.

66. Kundu, S.D., Roehl, K.A., Yu, X., et al. (2007) Prostate specific antigen density correlates with features of prostate cancer aggressiveness. J. Urol. 177, 505.

67. Radwan, M.H., Yan, Y., Luly, J.R., et al. (2007) Prostate-specific antigen density predicts adverse pathology and increased risk of biochemical failure. Urology 69, 1121.

68. Epstein, J.I., Walsh, P.C., Carmichael, M., et al. (1994) Pathologic and clinical findings to predict tumor extent of nonpalpable (stage T1c) prostate cancer. JAMA 271, 368. 
69. Tseng, K.S., Landis, P., Epstein, J.I., et al. (2010) Risk stratification of men choosing surveillance for low risk prostate cancer. J. Urol. 183, 1779.

70. Mikolajczyk, S.D., Millar, L.S., Wang, T.J., et al. (2000) "BPSA," a specific molecular form of free prostate-specific antigen, is found predominantly in the transition zone of patients with nodular benign prostatic hyperplasia. Urology $\mathbf{5 5}, 41$.

71. Linton, H.J., Marks, L.S., Millar, L.S., et al. (2003) Benign prostate-specific antigen (BPSA) in serum is increased in benign prostate disease. Clin. Chem. 49, 253.

72. Mikolajczyk, S.D., Grauer, L.S., Millar, L.S., et al. (1997) A precursor form of PSA (pPSA) is a component of the free PSA in prostate cancer serum. Urology 50, 710.

73. Peter, J., Unverzagt, C., Krogh, T.N., et al. (2001) Identification of precursor forms of free prostate-specific antigen in serum of prostate cancer patients by immunosorption and mass spectrometry. Cancer Res. 61, 957.

74. Mikolajczyk, S.D., Millar, L.S., Wang, T.J., et al. (2000) A precursor form of prostate-specific antigen is more highly elevated in prostate cancer compared with benign transition zone prostate tissue. Cancer Res. 60, 756.

75. Mikolajczyk, S.D. and Rittenhouse, H.G. (2003) Pro PSA: a more cancer specific form of prostate specific antigen for the early detection of prostate cancer. Keio J. Med. 52, 86.

76. Catalona, W.J., Bartsch, G., Rittenhouse, H.G., et al. (2003) Serum pro prostate specific antigen improves cancer detection compared to free and complexed prostate specific antigen in men with prostate specific antigen 2 to $4 \mathrm{ng} / \mathrm{ml}$. J. Urol. 170, 2181.

77. Sokoll, L.J., Chan, D.W., Mikolajczyk, S.D., et al. (2003) Proenzyme psa for the early detection of prostate cancer in the $2.5-4.0 \mathrm{ng} / \mathrm{ml}$ total psa range: preliminary analysis. Urology 61, 274.

78. Jansen, F.H., van Schaik, R.H., Kurstjens, J., et al. (2010) Prostate-specific antigen (PSA) isoform p2PSA in combination with total PSA and free PSA improves diagnostic accuracy in prostate cancer detection. Eur. Urol. [Epub ahead of print]

79. Sokoll, L.J., Wang, Y., Feng, Z., et al. (2008) [-2]Proenzyme prostate specific antigen for prostate cancer detection: a national cancer institute early detection research network validation study. J. Urol. 180, 539.

80. Le, B. V., Griffin, C. R., Loeb, S. et al. (2010) [-2]Proenzyme prostate specific antigen is more accurate than total and free prostate specific antigen in differentiating prostate cancer from benign disease in a prospective prostate cancer screening study. J. Urol. 183, 1355.

81. Catalona, W.J., Bartsch, G., Rittenhouse, H.G., et al. (2004) Serum pro-prostate specific antigen preferentially detects aggressive prostate cancers in men with 2 to $4 \mathrm{ng} / \mathrm{ml}$ prostate specific antigen. J. Urol. 171, 2239.

82. Makarov, D.V., Isharwal, S., Sokoll, L.J., et al. (2009) Pro-prostate-specific antigen measurements in serum and tissue are associated with treatment necessity among men enrolled in expectant management for prostate cancer. Clin. Cancer Res. 15, 7316.

83. Carter, H.B., Pearson, J.D., Metter, E.J., et al. (1992) Longitudinal evaluation of prostate-specific antigen levels in men with and without prostate disease. JAMA 267, 2215.

84. Carter, H.B. and Pearson, J.D. (1994) Evaluation of changes in PSA in the management of men with prostate cancer. Semin. Oncol. 21, 554.

85. Freedland, S.J., Humphreys, E.B., Mangold, L.A., et al. (2005) Risk of prostate cancer-specific mortality following biochemical recurrence after radical prostatectomy. JAMA 294, 433.

86. D'Amico, A.V., Cote, K., Loffredo, M., et al. (2003) Determinants of prostate cancer specific survival following radiation therapy during the prostate specific antigen era. J. Urol. 170, S42.

87. Loeb, S., Roehl, K.A., Helfand, B.T., et al. (2009) Can prostate specific antigen velocity thresholds decrease insignificant prostate cancer detection? J. Urol. 183, 112.

88. Carter, H.B., Ferrucci, L., Kettermann, A., et al. (2006) Detection of life-threatening prostate cancer with prostatespecific antigen velocity during a window of curability. J. Natl. Cancer Inst. 98, 1521.

89. D'Amico, A.V., Renshaw, A.A., Sussman, B., et al. (2005) Pretreatment PSA velocity and risk of death from prostate cancer following external beam radiation therapy. JAMA 294, 440.

90. D'Amico, A.V., Chen, M.H., Roehl, K.A., et al. (2004) Preoperative PSA velocity and the risk of death from prostate cancer after radical prostatectomy. N. Engl. J. Med. 351, 125.

91. Vickers, A.J., Savage, C., O'Brien, M.F., et al. (2009) Systematic review of pretreatment prostate-specific antigen velocity and doubling time as predictors for prostate cancer. J. Clin. Oncol. 27, 398.

92. Thompson, I.M., Ankerst, D.P., Chi, C., et al. (2006) Assessing prostate cancer risk: results from the Prostate Cancer Prevention Trial. J. Natl. Cancer Inst. 98, 529.

93. Spurgeon, S.E., Mongoue-Tchokote, S., Collins, L., et al. (2007) Assessment of prostate-specific antigen doubling time in prediction of prostate cancer on needle biopsy. Urology 69, 931.

94. Wolters, T., Roobol, M.J., Bangma, C.H., et al. (2009) Is prostate-specific antigen velocity selective for clinically significant prostate cancer in screening? European Randomized Study of Screening for Prostate Cancer (Rotterdam). Eur. Urol. 55, 385.

95. Carter, H.B., Partin, A.W., Luderer, A.A., et al. (1997) Percentage of free prostate-specific antigen in sera predicts aggressiveness of prostate cancer a decade before diagnosis. Urology 49, 379. 
96. O'Brien, M.F., Cronin, A.M., Fearn, P.A., et al. (2009) Pretreatment prostate-specific antigen (PSA) velocity and doubling time are associated with outcome but neither improves prediction of outcome beyond pretreatment PSA alone in patients treated with radical prostatectomy. J. Clin. Oncol. 27, 3591.

97. Vickers, A.J., Wolters, T., Savage, C.J., et al. (2009) Prostate-specific antigen velocity for early detection of prostate cancer: result from a large, representative, population-based cohort. Eur. Urol. [Epub ahead of print]

98. Carter, H.B., Pearson, J.D., Waclawiw, Z., et al. (1995) Prostate-specific antigen variability in men without prostate cancer: effect of sampling interval on prostate-specific antigen velocity. Urology 45, 591.

99. Kadmon, D., Weinberg, A.D., Williams, R.H., et al. (1996) Pitfalls in interpreting prostate specific antigen velocity. $J$. Urol. 155, 1655.

100. Loeb, S., Chan, D.W., Sokoll, L., et al. (2008) Prostate specific antigen assay standardization bias could affect clinical decision making. J. Urol. 180, 1959.

101. Loeb, S., Kettermann, A., Ferrucci, L., et al. (2008) The optimal application of prostate-specific antigen (PSA) velocity to predict high-risk disease. Eur. Urol. 54, 978.

102. Bussemakers, M.J., van Bokhoven, A., Verhaegh, G.W., et al. (1999) DD3: a new prostate-specific gene, highly overexpressed in prostate cancer. Cancer Res. 59, 5975.

103. de Kok, J.B., Verhaegh, G.W., Roelofs, R.W., et al. (2002) DD3(PCA3), a very sensitive and specific marker to detect prostate tumors. Cancer Res. 62, 2695.

104. Hessels, D., Klein Gunnewiek, J.M., van Oort, I., et al. (2003) DD3(PCA3)-based molecular urine analysis for the diagnosis of prostate cancer. Eur. Urol. 44, 8.

105. Marks, L.S., Fradet, Y., Deras, I.L., et al. (2007) PCA3 molecular urine assay for prostate cancer in men undergoing repeat biopsy. Urology 69, 532.

106. Whitman, E.J., Groskopf, J., Ali, A., et al. (2008) PCA3 score before radical prostatectomy predicts extracapsular extension and tumor volume. J. Urol. 180, 1975.

107. Nakanishi, H., Groskopf, J., Fritsche, H.A., et al. (2008) PCA3 molecular urine assay correlates with prostate cancer tumor volume: implication in selecting candidates for active surveillance. J. Urol. 179, 1804.

108. Haese, A., de la Taille, A., van Poppel, H., et al. (2008) Clinical utility of the PCA3 urine assay in European men scheduled for repeat biopsy. Eur. Urol. 54, 1081.

109. Tosoian, J.J., Loeb, S., Kettermann, A., et al. (2010) Accuracy of PCA3 measurement in predicting short-term biopsy progression in an active surveillance program. J. Urol. 183, 534.

110. Jakoby, W.B. (1978) The glutathione S-transferases: a group of multifunctional detoxification proteins. Adv. Enzymol. Relat. Areas Mol. Biol. 46, 383.

111. Lee, W.H., Morton, R.A., Epstein, J.I., et al. (1994) Cytidine methylation of regulatory sequences near the pi-class glutathione S-transferase gene accompanies human prostatic carcinogenesis. Proc. Natl. Acad. Sci. U. S. A. $91,11733$.

112. Nelson, W.G., De Marzo, A.M., Deweese, T.L., et al. (2001) Preneoplastic prostate lesions: an opportunity for prostate cancer prevention. Ann. N. Y. Acad. Sci. 952, 135.

113. Lin, X., Tascilar, M., Lee, W.H., et al. (2001) GSTP1 CpG island hypermethylation is responsible for the absence of GSTP1 expression in human prostate cancer cells. Am. J. Pathol. 159, 1815.

114. Brooks, J.D., Weinstein, M., Lin, X., et al. (1998) CG island methylation changes near the GSTP1 gene in prostatic intraepithelial neoplasia. Cancer Epidemiol. Biomarkers Prev. 7, 531.

115. Mao, L., Schoenberg, M.P., Scicchitano, M., et al. (1996) Molecular detection of primary bladder cancer by microsatellite analysis. Science 271, 659.

116. Goessl, C., Heicappell, R., Munker, R., et al. (1998) Microsatellite analysis of plasma DNA from patients with clear cell renal carcinoma. Cancer Res. 58, 4728.

117. Goessl, C., Krause, H., Muller, M., et al. (2000) Fluorescent methylation-specific polymerase chain reaction for DNA-based detection of prostate cancer in bodily fluids. Cancer Res. 60, 5941.

118. Sidransky, D. (1997) Nucleic acid-based methods for the detection of cancer. Science 278, 1054.

119. Cairns, P., Esteller, M., Herman, J.G., et al. (2001) Molecular detection of prostate cancer in urine by GSTP1 hypermethylation. Clin. Cancer Res. 7, 2727.

120. Goessl, C., Muller, M., Heicappell, R., et al. (2001) DNA-based detection of prostate cancer in urine after prostatic massage. Urology 58, 335.

121. Goessl, C., Muller, M., Heicappell, R., et al. (2001) DNA-based detection of prostate cancer in blood, urine, and ejaculates. Ann. N. Y. Acad. Sci. 945, 51.

122. Jeronimo, C., Usadel, H., Henrique, R., et al. (2002) Quantitative GSTP1 hypermethylation in bodily fluids of patients with prostate cancer. Urology 60, 1131.

123. Gonzalgo, M.L., Pavlovich, C.P., Lee, S.M., et al. (2003) Prostate cancer detection by GSTP1 methylation analysis of postbiopsy urine specimens. Clin. Cancer Res. 9, 2673.

124. Hoque, M.O., Topaloglu, O., Begum, S., et al. (2005) Quantitative methylation-specific polymerase chain reaction gene patterns in urine sediment distinguish prostate cancer patients from control subjects. J. Clin. Oncol. $23,6569$.

125. Gardiner, R.A., Samaratunga, M.L., Gwynne, R.A., et al. (1996) Abnormal prostatic cells in ejaculates from men with prostatic cancer--a preliminary report. Br. J. Urol. 78, 414.

126. Xu, J., Stolk, J.A., Zhang, X., et al. (2000) Identification of differentially expressed genes in human prostate cancer using subtraction and microarray. Cancer Res. 60, 1677. 
127. Ferdinandusse, S., Denis, S., IJlst, L., et al. (2000) Subcellular localization and physiological role of alphamethylacyl-CoA racemase. J. Lipid Res. 41, 1890.

128. Chan, J.M., Stampfer, M.J., Ma, J., et al. (2001) Dairy products, calcium, and prostate cancer risk in the Physicians' Health Study. Am. J. Clin. Nutr. 74, 549.

129. Jiang, Z., Woda, B.A., Rock, K.L., et al. (2001) P504S: a new molecular marker for the detection of prostate carcinoma. Am. J. Surg. Pathol. 25, 1397.

130. Rubin, M.A., Zhou, M., Dhanasekaran, S.M., et al. (2002) alpha-Methylacyl coenzyme A racemase as a tissue biomarker for prostate cancer. JAMA 287, 1662.

131. Luo, J., Zha, S., Gage, W.R., et al. (2002) Alpha-methylacyl-CoA racemase: a new molecular marker for prostate cancer. Cancer Res. 62, 2220.

132. Evans, A.J. (2003) Alpha-methylacyl CoA racemase (P504S) overview and potential uses in diagnostic pathology as applied to prostate needle biopsies. J. Clin. Pathol. 56, 892.

133. Gudmundsson, J., Sulem, P., Manolescu, A., et al. (2007) Genome-wide association study identifies a second prostate cancer susceptibility variant at 8q24. Nat. Genet. 39, 631.

134. Zheng, S.L., Sun, J., Wiklund, F., et al. (2008) Cumulative association of five genetic variants with prostate cancer. $N$. Engl. J. Med. 358, 910.

135. Gudmundsson, J., Sulem, P., Gudbjartsson, D.F., et al. (2009) Genome-wide association and replication studies identify four variants associated with prostate cancer susceptibility. Nat. Genet. 41, 1122.

136. Eeles, R.A., Kote-Jarai, Z., Al Olama, A.A., et al. (2009) Identification of seven new prostate cancer susceptibility loci through a genome-wide association study. Nat. Genet. 41, 1116.

\section{This article should be cited as follows:}

Tosoian, J. and Loeb, S. (2010) PSA and beyond: the past, present, and future of investigative biomarkers for prostate cancer. TheScientificWorldJOURNAL: TSW Urology 10, 1919-1931. DOI 10.1100/tsw.2010.182. 

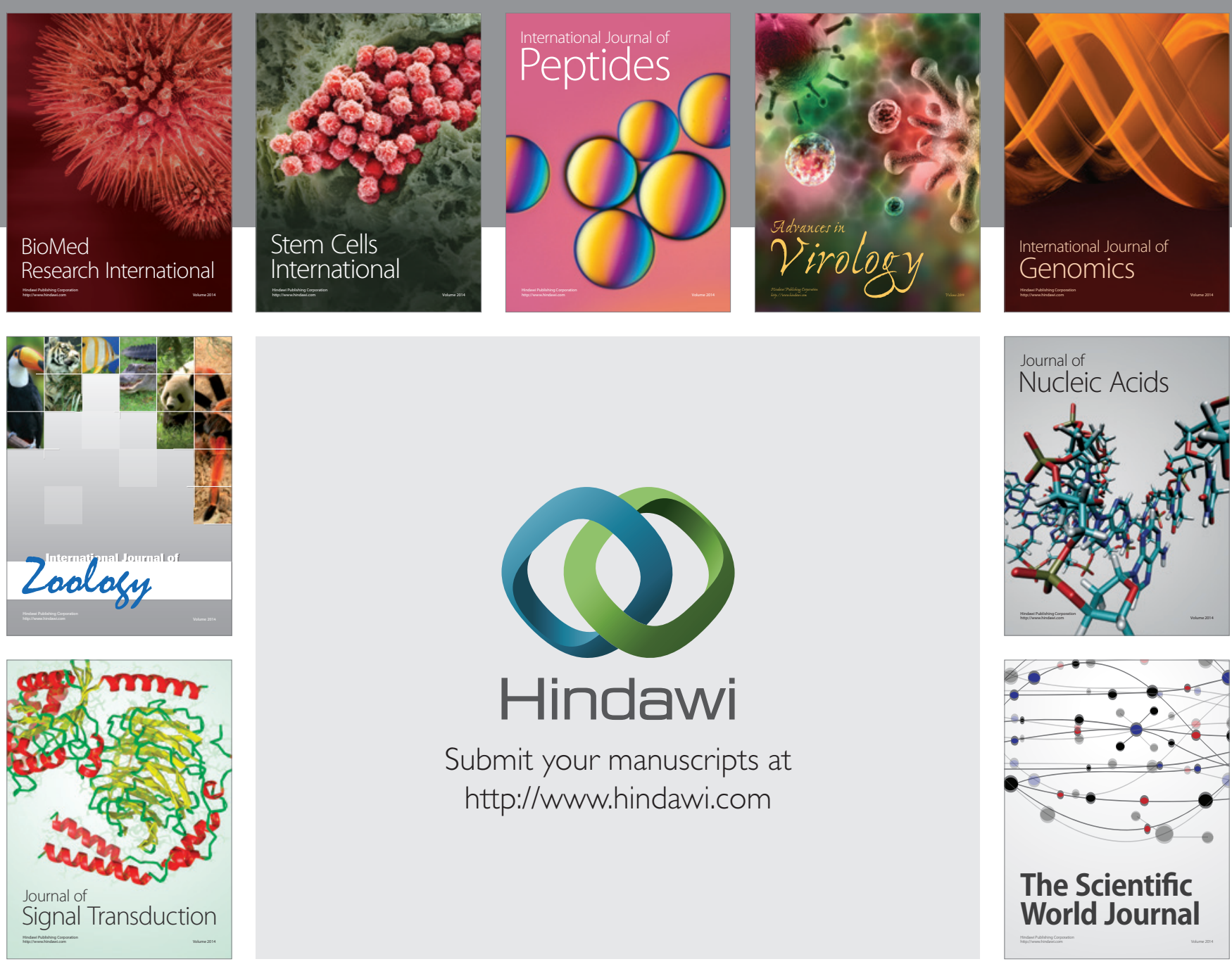

Submit your manuscripts at

http://www.hindawi.com
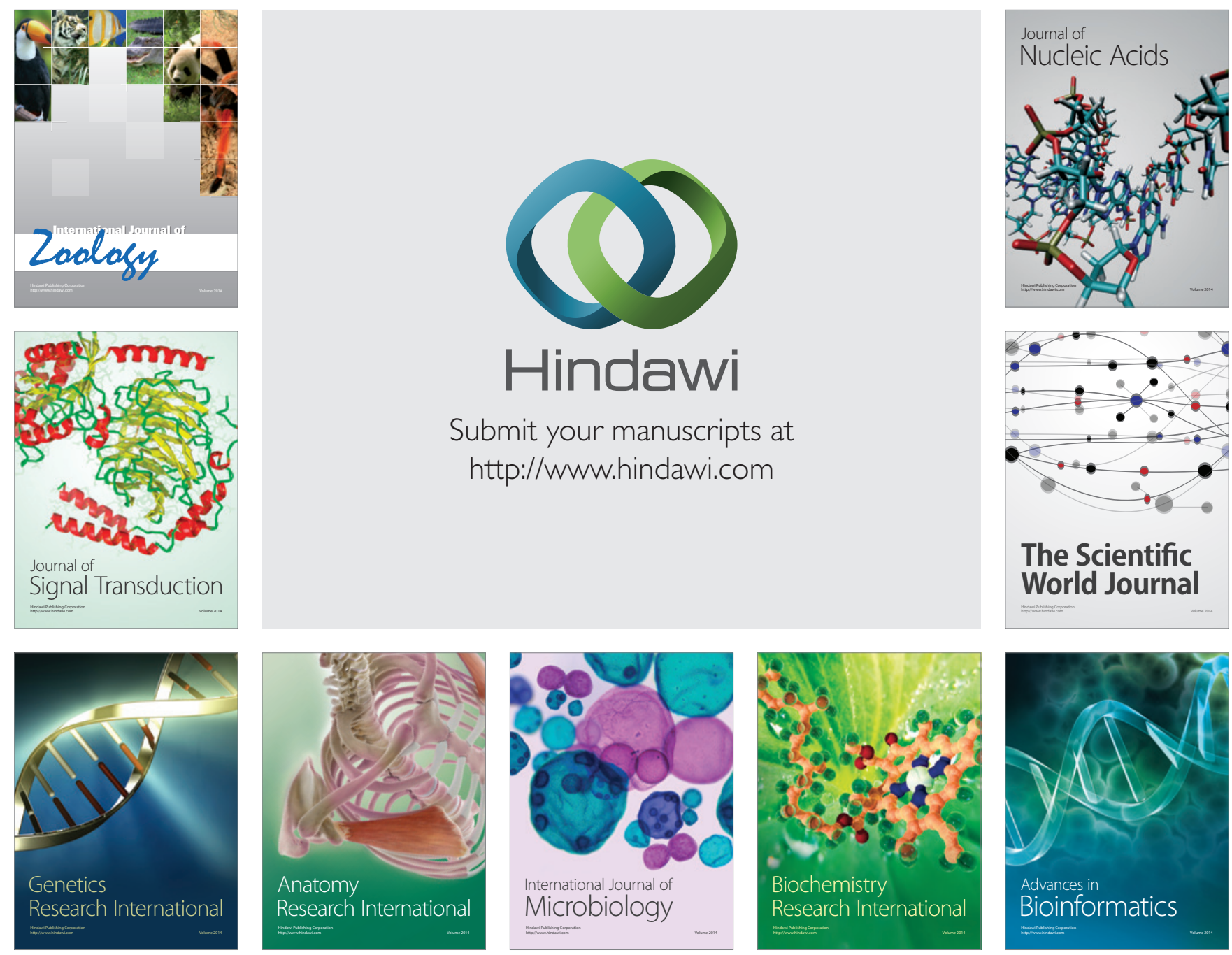

The Scientific World Journal
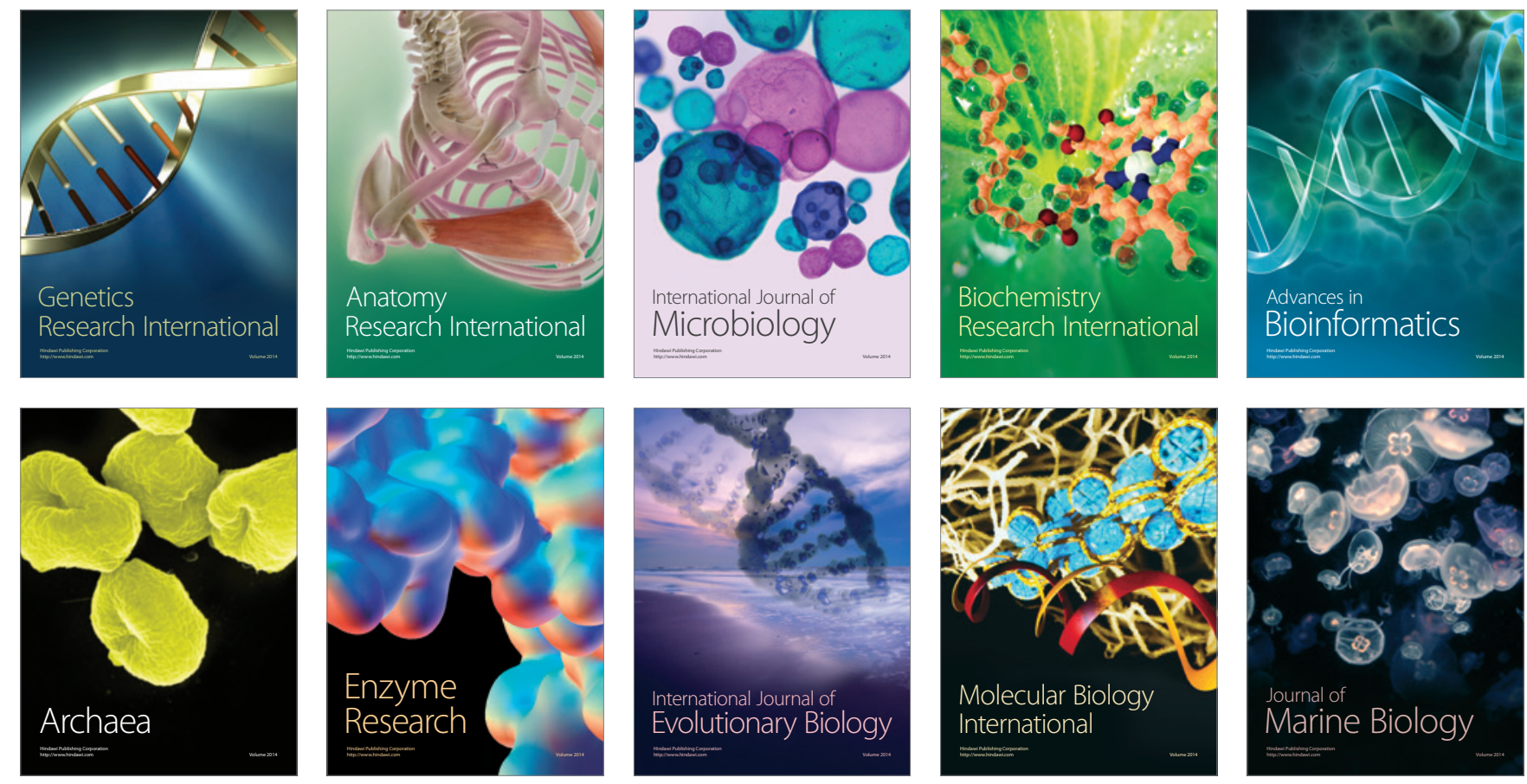